\title{
Combined Treatment of Disfiguring Facial Angiofibromas in Tuberous Sclerosis Complex With Surgical Debulking and Topical Sirolimus
}

\author{
Jessica L. Patterson, MD; Sanjana Iyengar, MD; Carson Catasus, BS; Joanna A. Kolodney, MD;
} Zachary Zinn, MD

Tuberous sclerosis complex (TSC) is an autosomal-dominant disorder that causes the formation of hamartomatous tumors such as facial angiofibromas (FAs). We present a combination of surgical debulking via shave biopsy, curettage, and electrocautery followed by application of sirolimus ointment $1 \%$ to the nose to treat FAs in the setting of TSC. This novel approach achieved an optimal therapeutic response in our patient with minimal recurrence of FAs after 1 year of follow-up.

Cutis. 2020;106:307-308.

\section{Practice Gap}

Tuberous sclerosis complex (TSC) is an autosomaldominant genetic disorder resulting in loss-of-function mutations in the TSC1 and TSC2 genes. These mutations lead to constitutive activation of the mitogenic mTOR pathway and release of lymphangiogenic growth factors, causing the formation of hamartomatous tumors throughout multiple organ systems. ${ }^{1}$ Facial angiofibromas (FAs) are a common cutaneous manifestation of TSC, affecting up to $80 \%$ of patients worldwide. ${ }^{2}$ Aesthetic disfigurement, vision obstruction, and breathing impairment often are associated with FAs. They frequently arise in children with TSC and impose a psychosocial burden that can affect the patient's overall quality of life.

Cutaneous stigmata of TSC pose a significant therapeutic challenge. Topical sirolimus has become a firstline treatment of FAs by inhibiting the mitogenic mTOR pathway'; however, thicker, more extensive lesions are less responsive to topical therapy. The entire dermis is involved in TSC, and topical sirolimus alone often is ineffective for large fibrous FAs. ${ }^{3}$ Likewise, oral mTOR inhibition has shown only $25 \%$ to $50 \%$ improvement in FAs and has potential side effects that can limit patients' tolerance and compliance. ${ }^{4}$

\section{The Technique}

A 46-year-old man with TSC was referred to dermatology for treatment of numerous facial papules and plaques that had been present since childhood and were consistent with FAs (Figure 1A). The lesions were tender, impaired the patient's breathing, and caused emotional distress. Dermabrasion was attempted 20 years prior with minimal improvement and subsequent progression of the FAs. Other stigmata of TSC were present, including cutaneous hypopigmented macules and shagreen patches as well as seizures and renal angiomyolipomas. Due to multiorgan involvement, the patient was started on once-daily oral everolimus $2.5 \mathrm{mg}$; however, the FAs were progressive despite the systemic mTOR inhibition. Furthermore, it was presumed that topical sirolimus monotherapy would be ineffective due to thickness and extent of FAs; therefore, we proposed a novel treatment approach combining initial surgical debulking with subsequent longitudinal use of topical sirolimus to reduce the risk of recurrence.

Local anesthesia with lidocaine $1 \%$ and epinephrine 1:100,000 was administered. Larger FAs were removed at the base with a sterile surgical blade. Nasal recontouring

From West Virginia University, Morgantown. Drs. Patterson, Iyengar, and Zinn are from the Department of Dermatology. Ms. Catasus is from the School of Medicine. Dr. Kolodney is from the Department of Hematology and Oncology. 
FIGURE 1. A and B, Multiple facial angiofibromas on the nose, cheeks, and nasolabial folds causing considerable nasal deformity and breathing impairment in a patient with tuberous sclerosis complex at presentation and immediately after surgical debulking, nasal recontouring, and extensive electrocautery for hemostasis.

FIGURE 2. A, Considerable cosmetic improvement and resolution of breathing impairment was noted 8 weeks following treatment. B, At 1-year followup, the patient demonstrated sustained clearance of facial angiofibromas on topical sirolimus monotherapy.
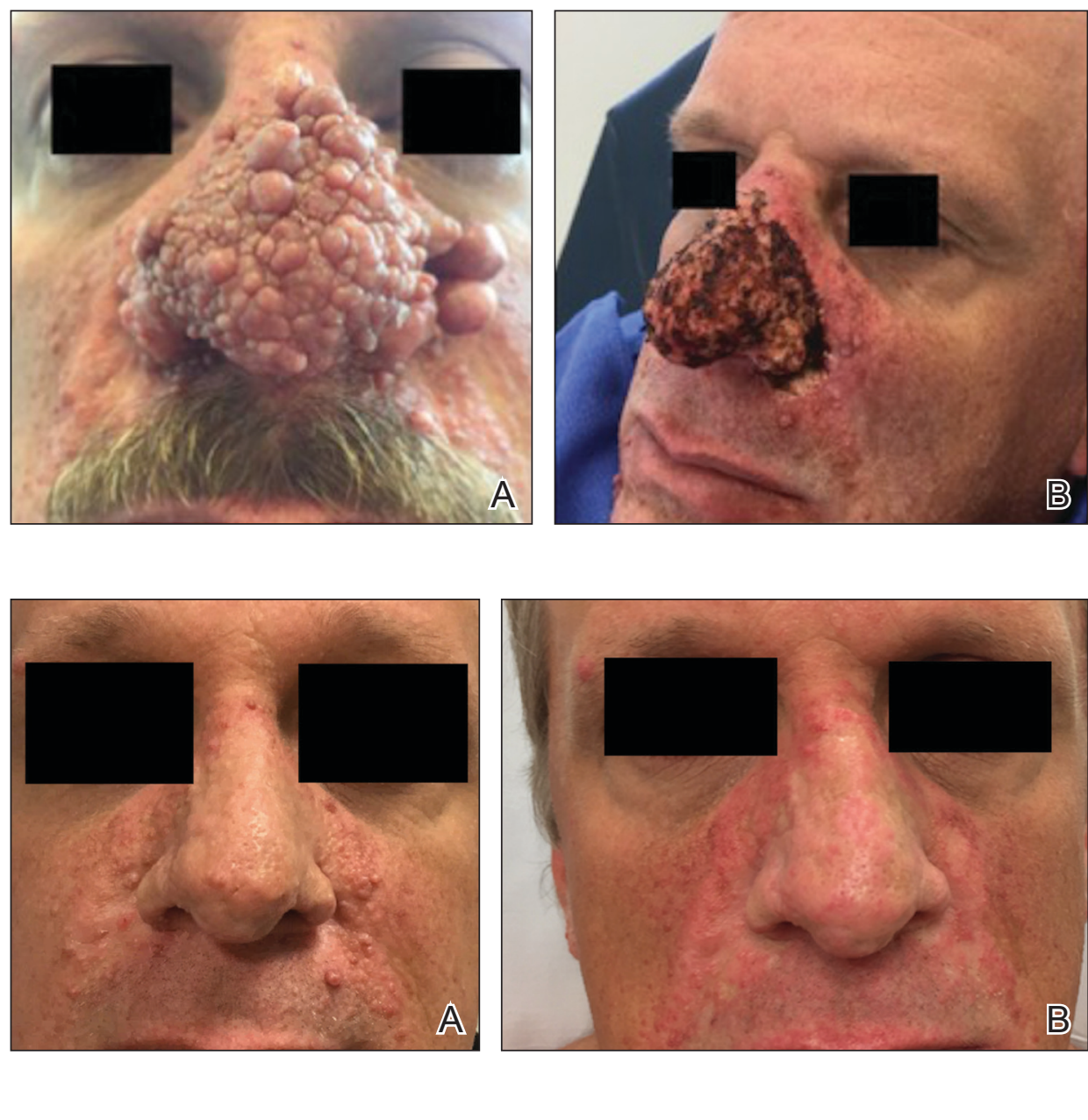

subsequently was performed using a combination of shave biopsy and curettage. Extensive electrocautery was performed for hemostasis and destruction of residual FAs. Figure $1 \mathrm{~B}$ shows the immediate postoperative result.

One month postoperatively, the patient stopped the oral everolimus at his oncologist's recommendation due to abdominal pain and peripheral edema. Once the abraded skin showed evidence of wound healing, the patient was instructed to initiate sirolimus ointment $1 \%$ twice daily to reduce the risk of recurrence. ${ }^{1,5,6}$ At 8-week follow-up, the patient was noted to have cosmetic improvement and resolution of breathing impairment (Figure 2A). He continued to show excellent cosmetic results at 1 -year follow-up using topical sirolimus monotherapy (Figure 2B).

\section{Practical Implications}

Surgical debulking combined with longitudinal use of sirolimus ointment $1 \%$ can achieve an optimal therapeutic response for disfiguring phymatous presentation of FAs in the setting of TSC. We believe it is an effective approach for thick disfiguring FAs that are unlikely to respond to $\mathrm{mTOR}$ inhibition alone.

\section{REFERENCES}

1. Wataya-Kaneda M, Nakamura A, Tanaka M, et al. Efficacy and safety of topical sirolimus therapy for facial angiofibromas in the tuberous sclerosis complex: a randomized clinical trial. JAMA Dermatol. 2017;153:39-48.

2. Koenig MK, Hebert AA, Roberson J, et al. Topical rapamycin therapy to alleviate the cutaneous manifestations of tuberous sclerosis complex. Drugs R D. 2012;12:121-126.

3. Wataya-Kaneda M, Ohno Y, Fujita Y, et al. Sirolimus gel treatment vs placebo for facial angiofibromas in patients with tuberous sclerosis complex: a randomized clinical trial. JAMA Dermatol. 2018;154:781-788.

4. Nathan N, Wang JA, Li S, et al. Improvement of tuberous sclerosis complex (TSC) skin tumors during long-term treatment with oral sirolimus. J Am Acad Dermatol. 2015;73:802-808.

5. Kaplan B, QaziY, Wellen JR. Strategies for the management of adverse events associated with mTOR inhibitors. Transplant Rev (Orlando). 2014;28:126-133.

6. Haemel AK, O’Brian AL, Teng JM. Topical rapamycin therapy to alleviate the cutaneous manifestations of tuberous sclerosis complex. Arch Dermatol. 2010;146:1538-3652. 